\title{
A Method to Detect Circle based on Hough Transform Mengjie $\mathrm{Wu}^{1,2, \mathrm{a}}$, Zongxi Song ${ }^{1}$, Baopeng $\mathrm{Li}^{1}$, Feipeng $\mathrm{Li}^{1,2}$, Bin $\mathrm{Li}^{1,2}$ and Chao \\ ${ }^{1}$ Xi'an Institute of Optics and Precision Mechanics of CAS, Xi'an, China \\ ${ }^{2}$ University of Chinese Academy of Sciences, Beijing, China \\ awumengjie@opt.cn
}

Keywords: Hough transform; Circle; cooperative target; effective area.

\begin{abstract}
During the butting procedure of the cooperative target, the ability that circle were detected accurately in complex background appears to be very important. In this paper, in order to overcome the ergodic of standard Hough transform, an improved HT of circle detection based on locating the effective area is carried out, which could wipe off many noneffective spots. Then, sampling the edge image makes further improvement in computing speed. The experiment results demonstrate that the method is less time-consuming compared with standard HT.
\end{abstract}

\section{Introduction}

The information of the circle target varies with the change of vision angle, range, illumination environment and so on. The standard method finding circle based on Hough transform has the advantages of strong noise resistance, strong robustness to interference point, low sensitivity to edge defect, excellent adaptability for parallel algorithms [1]. However, huge computation [2] and large time-consuming are the main limitations of the standard HT. In addition, the 3D projections of edge image in parameter space produce much errors, such as useless accumulation, quantization error [3]. Obvious, the standard HT can`t meet requirements of timeliness and veracity when the image background is too complex.

The paper is organized as follows. In the next section we introduce the basic idea underlying the HT. This is followed by a description in detail of the improved method detecting circle, which consists of image preprocessing, locating the effective area, HT computation and removal of incorrect detecting edge point. Next, the experimental evaluation is then given and the final section presents the conclusions of the work.

\section{Basic Idea of Hough Transform}

The idea of HT is shown as follows [4]. A circle in the image is described as Eq.1.

$$
\left(x-a_{0}\right)^{2}+\left(y-b_{0}\right)^{2}=r_{0}^{2}
$$

Where $(\mathrm{a}, \mathrm{b})$ are the coordinate of the circle center and $\mathrm{r}$ is its radius, then an arbitrary edge point $\left(x_{i}, y_{i}\right)$ will be transformed into a circular cone in the $(\mathrm{a}, \mathrm{b}, \mathrm{r})$ parameter space [5]. The circular cone can be defined as Eq.2.

$$
\left(a-x_{i}\right)^{2}+\left(b-y_{i}\right)^{2}=r^{2}
$$

The attractions of HT in computation are that it divide the parameters space abr into accumulation cells [6]. When the circular cones get through some cells, its accumulated value add one. As a result, the coordinate $\left(\mathrm{a}_{0}, b_{0}, r_{0}\right)$ of accumulation cell where the peak point is detected corresponds to the circle center and radius in image space.

\section{Image Preprocessing}

Hough transform is main applied to binary image so that it is necessary to do preprocessing for the original image [7], whose eventually results affect directly the circle detecting. A median filter 
and image contrast amplification were brought into the original image.

The logarithms \& image contrast stretching transformation is a useful tools to adjust dynamic range of image brightness [6], which is defined as Eq.3.

$$
g=c \times \log (1+f)
$$

Where $\mathrm{c}$ is a constant coefficient and $\mathrm{f}$ is the original image, then $\mathrm{g}$ is the export image. A major function of the equation is to compress dynamic range of image brightness. Those image gray level which is less than threshold value $M$ or larger would be compressed into a small range, resulting in higher image brightness contrast ratio [6].

\section{Locating Effective Area}

Those edge points which are not associated with the cooperative target increase calculation amount of the finding circle remarkably. The prior information, the edge curve of top left corner and top right corner presents 90 degrees approximately, can be used to locating the effective area. The result of searching right angles is shown in Fig.1. The procedures how to locate the effective area were listed as follows.

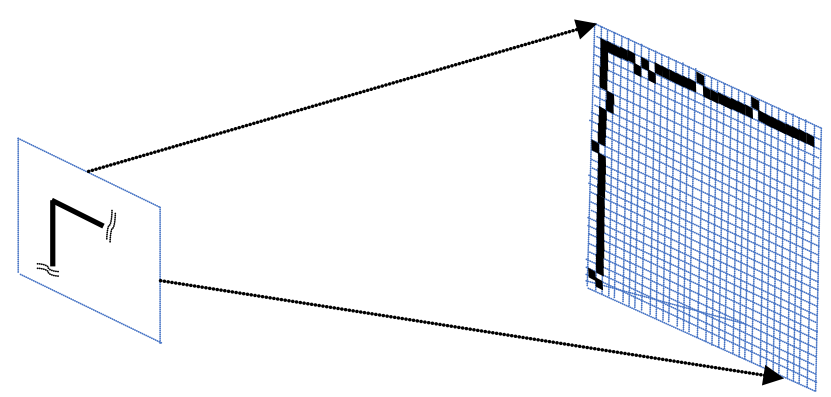

Fig.1, the result of searching right angles

I.A grid, whose scale is $30 \times 30$, starts to move from the top left point and the number of edge points in the grid were counted as count 0 . If count $0 \geq m$ and count $0 \leq n$, jump to step 2;

II.The distribution of count 0 points was detected to judge whether they present two segments and the width hold no more than 3 pixels. Then the angle between two segments is to be determined whether a right angle. If the answer is yes, jump to step 3. Otherwise, jump to step 1.

III.The grid continues to search along y direction and the number of edge points is counted as count 1 . If count $1 \geq m$ and count $1 \leq n$, jump to step 4 . Or, continue to search along y direction.

IV.The distribution of count1 points was detected to judge whether they present two segments and the width hold no more than 3 pixels. Then the angle between two segments is to be determined whether a right angle. If the answer is yes, jump to step 5. Otherwise, jump to step 3.

V. Setting the two break points of right angles which were detected in step 2 and 4 as features points. According to the prior information of the cooperative target, the effective area was located.

\section{Detecting Circle Based on HT}

On the premise that the number of effective edge points is enough to be detected, the edge image is sampled by a $75 \%$ ratio so that the speed of HT rises once more again. From the Eq. 1 above, the variables $a, b$ can be calculated by Eq.4.

$$
\left\{\begin{array}{l}
a=x_{i}+r \times \cos \theta \\
b=y_{i}+r \times \sin \theta
\end{array}\right.
$$

Standard HT traverses all the edge points, which means that double circulation by for-cycle computation is essential to transform from image space xoy to parameter space $a b r$. The matrix computation is regarded as a valid alternative to for-cycle. This transformation is defined as Eq.5. 


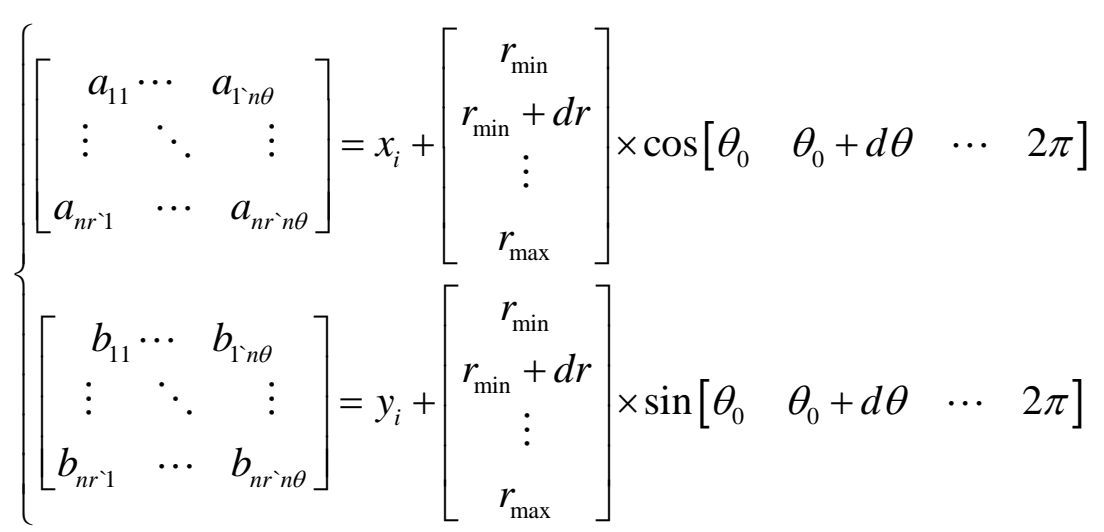

It is no doubt that some incorrect detecting edge points were detected as circular edge points because they possess some circular features, but not all. In order to enhance the experiment accuracy, some post processing procedures were taken to remove incorrect detecting edge points according to the prior information of cooperative target.

\section{Experimental Evaluation}

In the experiment, the system host is PC. CPU version is Inter Xeon. Master frequency is $3.7 \mathrm{GHz}$. RAM size is 4G. Simulation environment is Matlab. An original image, whose resolution ratio is $1051 \times 885$, is selected as experiment subject. The experiment results were shown in Fig.2.

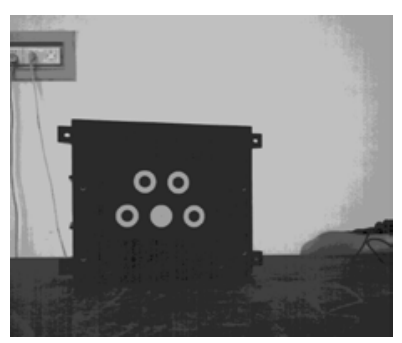

(a) original image

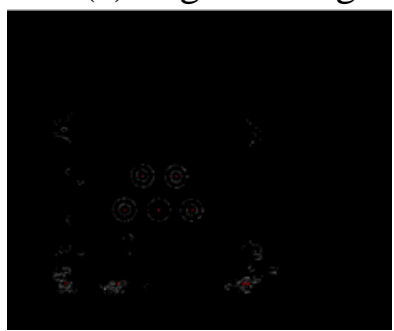

(d) detected circle

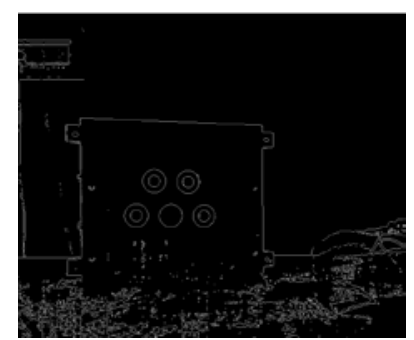

(b) edge image

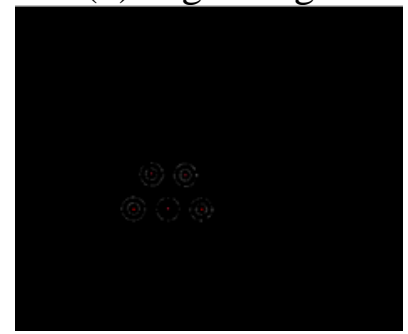

(e) removing errors

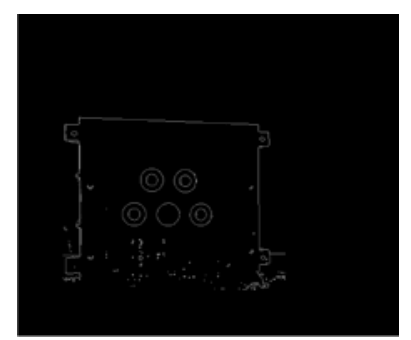

(c) effective area

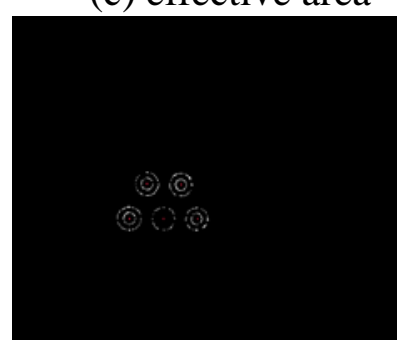

(f) image dilation

Fig.2, the experiment result

In the experiment, the standard finding circle method based on HT and the improved method were compared on time consuming and accuracy rate, which are shown separately in Table.1 and Fig.3.

Table 1, time consuming comparing

\begin{tabular}{ccccc}
\hline Original image & num of edge points & $t$ for sHT (s) & $t$ for new HT (s) & result \\
\hline Image 1 & 11032 & 22.52 & $9.04 \mathrm{~s}$ & $249.1 \%$ \\
\hline Image 2 & 12870 & 26.85 & $11.94 \mathrm{~s}$ & $224.9 \%$ \\
\hline
\end{tabular}




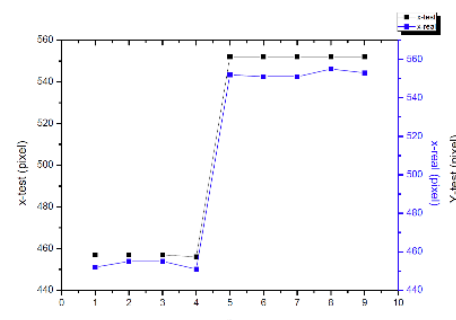

(a) x comparing

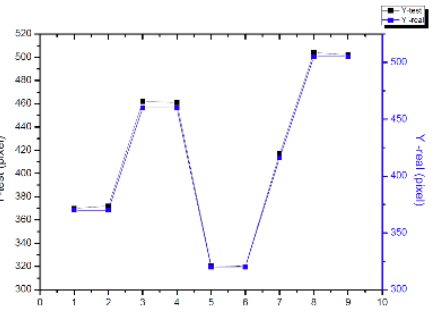

(b) y comparing

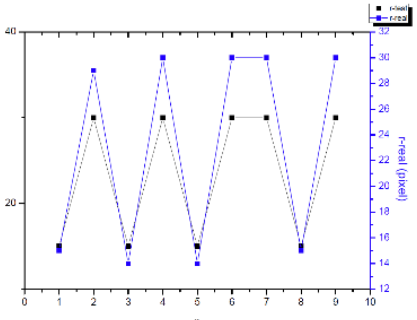

(c) r comparing

Fig.3, the accuracy comparing

\section{Summary}

In the paper, the new method not only takes possession of the inherent advantages of standard HT, such as strong noise resistance, strong robustness to interference point, low sensitivity to local edge defect but also improves the computation speed, which accelerates more than two times. What is more, it can be also applied to detecting different circle, whose radius and center are different completely, and the error rate between test value and real value is up to 95\%. Thus it can be seen that the new method detecting circle based on standard HT is feasible in real application and shows excellent computation performance.

\section{References}

[1] XIA Lei, CAI Chao, A new fast algorithm of Hough transform on detection of circles, Application Research of Computers. 24(10) 197-210.

[2] WANG Mian, ZHAO Jing xiu, The Research of Eye location Based on quick Hough Transformation, Technology Research. 11(8) 60-62.

[3] XU L, OJA E, Randomized Hough transform: basic mechanisms, algorithms, and computational complexities, Computer Vision Graphics Image Process: Image Understanding. 57(2) 131-154.

[4] OUGH P V C, U.S. Patent 3,069,645. (1962)

[5] Duda R.O., Hart P.E, Use of the Hough Transformation to detect lines and curves in pictures, Comm of the ACM.15(1) 11-15.

[6] Rafael C. Gonzalez, Richard E. Woods, Steven L. Eddins, Digital Image Processing using MATLAB, Electronics Industry, Bei Jing, 2005.

[7] YANG Quan yin, Shape Feature detection Based on Hough Transform in Image, Shandong University, Ji Nan, 2009. 